
\title{
25 Research Sourere \\ Up-regulated miR-224-5p can target the expression of Neuritin in hearing loss
}

\section{Yin Song}

Jiaxing Municipal Center for Disease control and Prevention

Xue Zhang

Tongxiang Center for Disease Control and Prevention

Jiawei Sun

Shihezi University School of Medicine

\section{Lina Li}

Jiaxing Municipal Center for Disease control and Prevention

Xiaofei Zhang

Jiaxing Municipal Center for Disease control and Prevention

\section{Ping Li}

Jiaxing Municipal Center for Disease control and Prevention

Jin Huang ( 1337956403@qq.com )

Shihezi University School of Medicine

\section{Yong Yan}

Jiaxing Municipal Center for Disease control and Prevention

\section{Research Article}

Keywords: Neuritin, Hearing loss, miR-224-5p, Bioinformatics, Sequencing

Posted Date: March 8th, 2022

DOI: https://doi.org/10.21203/rs.3.rs-1038261/v2

License: (c) (1) This work is licensed under a Creative Commons Attribution 4.0 International License.

Read Full License 


\section{Abstract \\ Objective}

Hearing loss is a common neurodegenerative disease and few studies on miRNAs associated with hearing loss. This study screened the differentially expressed miRNAs in hearing loss and explored the relationship between Neuritin and hearing loss at the same time.

\section{Methods}

The combination of kanamycin sulfate and furosemide was used to establish a mouse hearing loss model. High-throughput sequencing was used to screen the differentially expressed miRNAs during hearing loss. ABR was used to detect listening function. QRT-PCR was used to identify the expression of differential miRNAs in hearing loss. Western blot was used to detect the expression of Neuritin protein. Luciferase was used to identify the binding sites of miRNA and Neuritin.

\section{Results}

Neuritin expression decreases after hearing loss. 24 up-regulated miRNAs and 66 down-regulated miRNAs were screened by sequencing. The expression of miR-224-5p increased in the Corti after hearing loss $(p<0.05)$. MiR-224-5p can inhibit expression of Neuritin and specifically bind to neuritin gene $(p<$ $0.05)$.

\section{Conclusion}

Up-regulated miRNA-224-5p in hearing loss can target the expression of Neuritin.

\section{Introduction}

Hearing loss is a common disturbance of sensation and cause a significant disease burden ${ }^{1}$. According to WHO reports, the global number of disability deafness reached 466 million in 2019, and expected to exceed 900 million by 2050. Among people over 65 years old, one-third suffer from hearing loss. Hearing loss not only affects communication, but also related to Alzheimer's ${ }^{2}$ and mental illness ${ }^{3}$. The number of hearing loss is increasing continuously.

Sensorineural hearing loss (SNHL) occurs after the sensory hair cells or nerve structure of the cochlea damaged, which accounts for the majority of hearing loss ${ }^{4}$. The main causes of hearing loss included aging, genetic mutations, noise exposure, exposure to therapeutic drugs with ototoxic side effects, and chronic diseases ${ }^{5}$. Treatment for sensory hearing loss mainly relies on cochlear implant, requiring patient still have some functional hair cells and spiral neurons $s^{6,7}$. Hearing loss research mainly focuses on the 
local delivery of drugs to the cochlea ${ }^{8}$, gene therapy ${ }^{9}$ and cell therapy ${ }^{10}$. However, no drug-based therapy for hearing loss has been approved by the Food and Drug Administration, and treatment mostly relies on hearing aids or cochlear implant.

Delivering neurotrophic factors of the cochlea is a promising way to treat hearing loss. Brain-derived neurotrophic factor (BNDF) and neurotrophins-3 (NT-3) have been proof can promote the synaptic regeneration of SGN peripheral fibers in the hair cell and save the hearing function of adult animals after acoustic trauma ${ }^{11-13}$. Neuritin is a neurotrophic factor closely related to neuroplasticity, neurites growth and synapses maturity ${ }^{14}$. Neuritin can regulate the formation of synaptic circuits ${ }^{15}$, inhibit apoptosis, and maintain the survival of neurons ${ }^{16}$. Participating in the development of central nervous system, Neuritin also related to nerve regeneration, repair, learning and memory ${ }^{17}$. A new study shows that Neuritin is associated with hearing loss ${ }^{18}$.

MiRNA is a set of short non-coding RNAs that help regulated gene expression ${ }^{19}$. Since 1993, many miRNAs have been discovered in inner and outer ears playing a role in the normal development ${ }^{20,21}$. As most protein-coding genes in vertebrates are regulated by miRNA, the ability of miRNA to regulate mRNA in the auditory system is worthy of further study 22,23 .

As a neurotrophic factor, Neuritin has shown a correlation between hearing loss. This paper mainly discusses the relationship between Neuritin and hearing loss, and explores miRNAs related to hearing loss.

\section{Results}

\section{Hearing loss model construction and identification}

Hearing loss models to build by injecting with kanamycin sulfate and furosemide in combination and hearing detected by using $A B R{ }^{24,25}$. The disappearance of the second wave of $A B R$ waveform as the basis of determining the hearing threshold of mouse. According to the result, the normal hearing threshold was 15-20dB. Compared with control group, hearing loss group's ABR wave disappears in the 20-70 dB stage (Fig.1A), and the hearing threshold rises from 20-30dB to 70-80dB ( $<<0.05)$ (Fig.1B). After injected with kanamycin sulfate and furosemide, the hearing hearing loss $12 \mathrm{~h}$ and $24 \mathrm{~h}$ groups all had the same threshold rises. The hearing waveform and hearing threshold of the control group were still normal.

\section{The relationship between hearing loss and Neuritin}

After the hearing loss model was constructed, we detected the Neuritin protein expression of mouse ear by western blot (WB) at different times. Every 6 Corti were combined with a group of detection, and WB results show that the expression of Neuritin decreased significantly after hearing loss $(p<0.05)$ (Fig. $2 A)$. Compared with the $12 \mathrm{~h}$ group, the expression of Neuritin in the $24 \mathrm{~h}$ group recovered a little. Although there was a significant decrease in Neuritin expression, it was not statistically significant (Fig. 2B). 


\section{Analysis and screening of differentially expressed miRNAs}

Successful hearing loss models were selected for miRNA sequence. miRNA sequence was proceeded by RNA-seq after Corti RNA obtained from the mouse. The miRNA sequences were analyzed by DEG-seq, and screened in accordance with $|\log 2 \mathrm{FC}|>1$, Q.Value $<0.001$. In the hearing loss $12 \mathrm{~h}$ group, 80 significantly up-regulated miRNAs and 169 significantly down-regulated miRNAs were found (Fig. 3A-B). In the hearing loss $24 \mathrm{~h}$ group, 40 significantly up-regulated miRNAs and 94 significantly down-regulated miRNAs were found (Fig. 3C-D). After overlapping the up-miRNAs of the $12 \mathrm{~h}$ group and the $24 \mathrm{~h}$ group, 24 co-upregulated miRNAs were screened (Fig. 3E). Also overlapping down regulated miRNAs, 66 co-downregulated miRNAs were screened. (Fig. 3F). The details of screened miRNAs are put into Table1. The results of the sequencing analysis can be found in supplementary table s1.

\section{MiRNA profiling targeting Neuritin in hearing loss}

After obtaining the differentially expressed miRNAs, we searched for miRNAs targeting Neuritin by bioinformatics analysis. Seven bioinformatics websites (TargetScan, mRDB, DIANA, miRNAMap, miRWalK, miRmap, trabase) were used to predict miRNAs targeted Neuritin and 1607 miRNAs were found finally (Supplementary Table S2). Overlapping 1607 Neuritin-targeting miRNAs with 24 co-upregulated miRNAs, we finally screened 15 miRNAs including miR-6395, miR-211-5p, miR-196a-5p, miR3073a-5p, miR-133b-3p, miR-21a-3p, miR-1247-3p, miR-181a-2-3p, miR-224-5p, miR-133a-3p, miR-12475p, miR-145a-5p, miR-93-3p, miR-339-5p and miR-1198-5p (Fig. 4A).

\section{MiRNA-224-5p is upregulated in hearing loss and negative related to Neuritin}

By querying Pubmed, we found that miR-224 is associated with inflammation of the inner ear ${ }^{26}$, and multiple inner ear inflammations are associated with hearing loss ${ }^{27}$. The neck loop method was used to synthesize primers for miR-224-5p and U6 was used as an internal reference for QRT-PCR. The miR-224$5 p$ expression increased after hearing loss $12 \mathrm{~h}$ and $24 \mathrm{~h}$ when detected by QRT-PCR again $(\mathrm{P}<0.05)$ (Fig. $4 A)$. Over time, the expression of miR-224-5p decreased a little. We synthesized mimics of miRNA-224-5p and used WB to detect Neuritin expression after miRNA-224-5p mimics was transfect into 293T cells. The expression of Neuritin was reduced after transfection( $P<0.05)$ (Fig. 4B-C).

\section{miR-224-5p and Neuritn are specific binding}

Through the mir-map website, we predicted the binding site between miR-224-5p and Neuritin (Fig. 5A). We cloned the 60bp Neuritin sequences in the GP-miRGLO vector for dual luciferase experiments (Supplementary Figure F1). The Luciferase results showed that the Neuritin expression was decreased in the WT group after transfection GP-miRGLO vector and miR-224-5p mimics $(p<0.05)$ (Fig. 5D). And the Neuritin expression of the Control group and the MUT group were still normal.

\section{Discussion}


Our study identified a miRNA-Neuritin-hearing loss axis in which miR-224-5p was up-regulated to downregulate the expression of cochlear Neuritin and be involved in the occurrence of hearing loss. It may provide new insights into the occurrence of hearing loss.

There are few research data related to hearing loss and Neuritin. The current research data show that Neuritin was up-regulated in hearing loss mice, and may promoted neuronal survival and prolonged plasticity of the superior paraolivary nucleus circuitry ${ }^{28}$. Neuritin was also found to be a risk factor associated with hearing $\operatorname{loss}^{18}$. Although there were few studies on Neuritin, there were some studies on the same neurotrophic factor and hearing loss. According to reports, exogenous administration of neurotrophins, NT-3, and BNDF can protect hearing loss and hair cell damage ${ }^{11-13}$, proved that the deficiency of neurotrophic factor was one of the causes of hearing loss. Our results show that Neuritin in Corti was inversely associated with hearing loss, which indicated that the decline in Neuritn may be one of the reasons for the occurrence of hearing loss.

A number of microRNAs are known to be associated with hearing loss ${ }^{29}$, but there are few miRNA sequencing data associated with hearing loss. We obtained differentially expressed miRNAs by using next-generation sequencing in hearing loss model mice.

For a long time, it is a classic method of building sensorineural hearing loss models that combine amino glycosides and diuretics ${ }^{30,31}$. When amino glycosides and diuretics are used in combination, diuretics can promote the passage of amino glycosides through the blood-cochlear barrier ${ }^{32,33}$, change the lymphatic potential of the inner ear to make amino glycosides more effectively diffuse to the cochlea ${ }^{34,35}$, and reduce the renal clearance of kanamycin ${ }^{36}$. This is a rapid and violent deafness reaction and the cochlear out hair cells will be lost soon ${ }^{25}$. In order to detect miRNA changes of hair cells during hearing loss, we finally selected two time points of $12 \mathrm{~h}$ and $24 \mathrm{~h}$ for detection.

MiRNAs are small endogenous RNAs that can regulate gene-expression posttranscriptionally ${ }^{37}$. Neuritin was decreased in hearing loss, so we mainly focused on up-regulated miRNAs. We thought that the miRNAs that changed in both $12 \mathrm{~h}$ and $24 \mathrm{~h}$ were more representative, so we screened 24 up-regulated miRNAs in hearing loss. To find the relationship between the 24 up-regulated miRNAs and Neuritin, we used seven bioinformatics sites to predict miRNAs targeting Neuritin. We were fortunate to find 15 miRNAs that may target Neuritin and up-regulated in hearing loss including miR-6395, miR-211-5p, miR196a-5p, miR-3073a-5p, miR-133b-3p, miR-21a-3p, miR-1247-3p, miR-181a-2-3p, miR-224-5p, miR-133a3p, miR-1247-5p, miR-145a-5p, miR-93-3p, miR-339-5p and miR-1198-5p. Investigating the 15 miRNAs in pubmed, we found that miR-224-5p is involved in cochlear inflammation ${ }^{26}$, so we believe miR-224-5p involved in miRNA-Neuritin-hearing loss axis.

Our results demonstrate that the miR-224-5p-Neuritin-hearing loss axis exists truly. From Fig. 5 we can see that miR-224-5p in Corti was significantly upregulated after hearing loss, miR-224-5p can inhibition the expression of Neuritin, and the luciferase result proved that Neuritin specifically binds to miR-224-5p. So, miR-224-5p was involved in the occurrence of hearing loss by inhibiting the expression of Neuritin. 
Due to the lack of more experimental data, the effects of miR-224-5p and Neuriitn on hearing loss still need further study.

Our discovery suggests a new strategy for treating hearing loss. We can reduce the expression of miR224-5p in the cochlea to increase the expression of Neuritin, which may alleviate the occurrence of hearing loss. At the same time miR-224-5p also can be used as a new marker for hearing loss. In summary, we found a miR-224-5p-Neuritin-hearing loss axis, which may expose a new way of regulating hearing loss.

\section{Conclusion}

miR-224-5p is up-regulated in hearing loss and targets the expression of Neuritin.

\section{Methods}

All procedures were approved by the Animal Ethical and Welfare Ethics Committee of Hangzhou Normal University and were performed in accordance with relevant guidelines and regulations. All our animal experiments methods were in accordance with ARRIVE guidelines.

\section{Construction of hearing loss model}

Hearing loss model established by injecting kanamycin sulfate and furosemide. First, two-month-old CBA mouse (without gender distinction) was selected. Second, CBA mouse was given subcutaneous injection of kanamycin sulfate 1,000 mg $\mathrm{kg}^{-1}$ (Biosharp, China). Last, after half an hour, CBA mouse was given intraperitoneal injection with furosemide $500 \mathrm{mg} \cdot \mathrm{kg}^{-1}$ (Sigma, USA). In this experiment, the mice were divided into 3 groups, the first group was the control group $(n=27)$, the second group was the druginduced injury group of 12 hours ( $n=27)$, and the third group was the drug-induced injury group of 24 hours $(n=27)$. The animals used in this experiment were CBA mice (SPF grade), and all the experimental animals were purchased from the Experimental Animal Center of Hangzhou Normal University.

\section{Auditory Brainstem Response (ABR)}

All mice require ABR testing ( $n=81)$. Each CBA mouse was given intraperitoneal injection of sodium pentobarbital $50 \mathrm{mg} \cdot \mathrm{kg}^{-1}$ (assisted exit, Canada) and placed in a standard shielded soundproof room after anesthesia. The detection electrodes were placed behind the left and right auricles and under the scalp of the mouse to perform the auditory brainstem response of the mouse. The stimulus sound is a short stimulus, the scanning duration is $10 \mathrm{~ms}$, the stimulus repetition rate is 11 times $\cdot \mathrm{s}^{-1}$, the filter bandpass is $150-1500 \mathrm{~Hz}$, and the superimposition is 500-1 000 times. Each mouse was tested for binaural hearing, and the second wave threshold was marked as the mouse hearing threshold.

\section{Preparation of Corti RNA}


The cochlea was removed immediately after the mouse was sacrificed by cervical dislocation. Cochlea was transferred to a dissecting microscope to strip the outer cochlear bone structure and then got the Corti organ $(n=54)$. Every 6 Corti organs were combined with a set and grinded by liquid nitrogen, then transfer the powder to a $1.5 \mathrm{~mL}$ EP tube. $1 \mathrm{~mL}$ TRNzol Universal (TIANGEN, China) was added to the EP tube, mix well and let stand for 10 minutes. $200 \mathrm{uL}$ chloroform was added to the EP tube, shake vigorously for $30 \mathrm{~s}$ and then let it stand for 10 minutes. Centrifuge at $12000 \mathrm{rpm}$ for 15 minutes. The upper liquid was taken to a new 1.5ML EP tube and added $500 \mathrm{~mL}$ isopropanols. Mix the tube well and let it stand at room temperature for 10 minutes. Centrifuge at $12000 \mathrm{rpm}$ for 10 minutes. After discarding the supernatant, added $1 \mathrm{ML}$ of $75 \%$ ethanol in the tube and let stand at room temperature for 5 minutes. Centrifuge at 8000 rpm for 5 minutes. After discarding the supernatant, added 20ul RNase-free water and stored at $-80^{\circ} \mathrm{C}$.

\section{4. miRNA sequencing and bioinformatics analysis}

The RNA-seq method was used to perform miRNA sequence of sample RNA ( $n=27)$, and the sequence depth of each sample was $20 \mathrm{M}$. The DEG-seq method was used to analyze the miRNA sequences and screen the miRNAs with different expressions. The screening criteria are $|\log 2 \mathrm{FC}|>1$ and $\mathrm{Q}$. Value $<0.001$. "NRN1" is used as a keyword to predict the miRNA targeting Neuritin gene from 7 different websites (TargetScan, miRDB, DIANA, miRNAMap, miRWalK, miRmap, trabase). The miRNA that was up-regulated in the 12-hour group and the 24-hour group was selected as a candidate miRNA.

\section{5.q-PCR}

The stem-loop method was used to synthesize candidate miRNA primers, and U6 was used as an internal reference. According to Takara Reverse Transcription Kit (Takara, Japan) $10 \mu \mathrm{L}$ system (5×PrimeScript Buffer $2 \mu \mathrm{L}$, PrimeScript RT Enzyme Mix I $0.5 \mu \mathrm{L}$, PCR Reverse Primer $0.5 \mu \mathrm{L}$, Total RNA $2 \mu \mathrm{L}, \mathrm{ddH}_{2} \mathrm{O} 5 \mu \mathrm{L}$ ), the extracted RNA was reversing transcribed into cDNA $(n=9)$. The cycle conditions were: $95^{\circ} \mathrm{C} 30 \mathrm{~s}(1$ cycle), $95^{\circ} \mathrm{C} 5 \mathrm{~s}, 60^{\circ} \mathrm{C} 30 \mathrm{~s}$ ( 40 cycles). According to the Takara qPCR Kit (Takara, Japan) $20 \mu \mathrm{L}$ system (

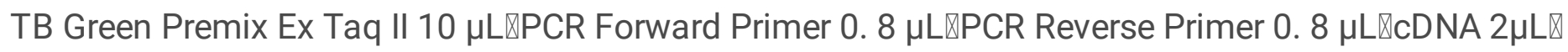
$\mathrm{ddH}_{2} \mathrm{O} 6.4 \mu \mathrm{L}$ ), the obtained cDNA was subjected to real-time fluorescence quantitative PCR, and the cycling conditions were $95^{\circ} \mathrm{C} 30 \mathrm{~s}(1 \mathrm{cycle}), 95^{\circ} \mathrm{C} 5 \mathrm{~s}$, and $60^{\circ} \mathrm{C} 30 \mathrm{~s}$ (40 cycles). Use the fluorescence quantitative PCR instrument (Germany analytik-jena 870 wer $3 \mathrm{G}$ ) to measure the fluorescence of the sample, and calculate the CT value and TM values. The $\triangle \triangle \mathrm{t}$ method was used to calculate the differential expression folds of candidate miRNAs between the $12 \mathrm{~h}$ group, $24 \mathrm{~h}$ group and the control group, and the target miRNAs were screened according to the differential fold.

The primers were designed by the Primer Premier 5.0 Software and as follows:

miR-224-5p-F: 5'- CGCGCGTAAGTCACTAGTGGT -3'

miR-224-5p-RT: 5'-GTCGTATCCAGTGCAGGGTCCGAGGTATTCGCACTGGATACGACAACGGA-3' 
U6-F: 5'-CTCGCTTCGGCAGCACA-3'

U6-R: 5'-AACGCTTCACGAATTTGCGT-3'

\section{Western blot}

The synthetic mimics of candidate miRNAs were transfected into 293T cells with Lipofectamine 3000 (ThermoFisher, USA), and after 48 hours of culture, the cells were lysed with RIPA (Beyotime, China) to extract total protein. ConFig.ure a $15 \%$ concentration SDS-PAGE gel, separate out $60-100$ ug of protein on the SDS-PAGE gel under the conditions of $80 \mathrm{~V}$ for 30 mins and $110 \mathrm{~V}$ for $90 \mathrm{mins}$, and use the semi-dry transfer method to transfer the protein under the conditions of $23 \mathrm{~V}$ for $43 \mathrm{mins}$. Onto PVDF membrane (Immobilon, ISEQ00010). Use 5\% skimmed milk powder (Biosharp, BS 102) to seal the PVDF membrane for $2 \mathrm{hs}$, then use 5\% skimmed milk powder to dilute Neuritin antibody (Abcam, 64186) at a ratio of 1:1000, and incubate overnight at $4^{\circ} \mathrm{C}$. On the next day, the goat anti-rabbit secondary antibody (Nakayama Jinqiao) was diluted at a ratio of 1:10000, incubated at room temperature for 2 hours, and an enhanced chemiluminescence system (BIO-RAD, 1705060) was used to detect the immune response zone.

\section{Luciferase}

Neuritn and miR-224-5P binding sites were predicted through miRmap (https://mirmap.ezlab.org/). Neuritn sequences in a length of 60 bp were cloned into the GP-miRGLO vector. GP-miRGLO vector and miR-224-5P mimics rely on Lipofectamine 3000 (Thermofisher, L3000015) to be transfected into 293T cells. After 48 hours of transfection, the lysate was collected, and the phochillase report system (DualLuciferase ${ }^{\circledR}$ Reporter (DLR ${ }^{\mathrm{TM}}$ ) Assay System, E1980) was used to measure the photinus pyralis luciferase and Renilla ReniFormis luciferase activity. Computational fluorescence activity ratio of firefly and sea kidney fluorescence activity. Three biological repeats were performed.

The Neuritn sequences were cloned into the GP-miRGLO vector as follows:

NRN1 -miR-224-5p WT:

GAGAGGGAAAAGGAGAAGGCCAGGGGAATGACTTCAAGAGTGGTGTCCACGTGGGAATCA

NRN1 -miR-224-5p MUT:

GAGAGGGAAAAGGAGAAGGCCAGGGGAAACTGAACAAGAGTGGTGTCCACGTGGGAATCA

\section{Declarations}

\section{Data availability:}

The data used and/or analyzed during the present study are available from the corresponding author on reasonable request.

\section{Acknowledgments}


The authors would like to thank the Animal Center of Hangzhou Normal University for breeding the mice. Dr LY (Department of Prevention Medicine, School of Medicine, Hangzhou Normal University, Hangzhou, Zhejiang 310036, P.R. China) kindly provided the experimental equipment.

\section{Author contributions}

J.H. contributed to the conception or design of the study. Y.S., X.Z. and JS contributed to the acquisition, analysis, or interpretation of all cell-level data. Y.S. contributed to the acquisition, analyze and interpretation of the bioinformatics data. Y.S., Y.Y., L.L., P.L., X.Z. and J.H. contributed to the drafting of the article and revising it critically for all content. All authors contributed to revising the work critically for important intellectual content.

\section{Funding}

The present study was supported by the Zhejiang Provincial Natural Science Foundation of China (grant no. $\mathrm{LY} 18 \mathrm{H} 260002$ to $\mathrm{YH}$ ).

\section{Competing interests}

The authors declare that they have no competing interests.

\section{Ethics declarations}

All our animal experiments meet the requirements of animal ethics and are supervised by the Animal Ethical and Welfare Ethics Committee of Hangzhou Normal University. Our research accord to the ARRIVE guidelines (PLoS Bio 8(6), e1000412,2010).

\section{References}

1. Karikari, T. K., Charway-Felli, A., Hoglund, K., Blennow, K. \& Zetterberg, H. Commentary: Global, regional, and national burden of neurological disorders during 1990-2015: a systematic analysis for the Global Burden of Disease Study 2015. Front Neuro/ 9, 201, doi:10.3389/fneur.2018.00201 (2018).

2. Ray, M., Dening, T. \& Crosbie, B. Dementia and hearing loss: A narrative review. Maturitas 128, 64-69, doi:10.1016/j.maturitas.2019.08.001 (2019).

3. Blazer, D. G. \& Tucci, D. L. Hearing loss and psychiatric disorders: a review. Psychol Med 49, 891-897, doi:10.1017/S0033291718003409 (2019).

4. Walling, A. D. \& Dickson, G. M. Hearing loss in older adults. Am Fam Physician 85, 1150-1156 (2012).

5. Cunningham, L. L. \& Tucci, D. L. Hearing Loss in Adults. N Engl J Med 377, 2465-2473, doi:10.1056/NEJMra1616601 (2017).

6. Pfingst, B. E. et al. Cochlear infrastructure for electrical hearing. Hear Res 281, 65-73, doi:10.1016/j.heares.2011.05.002 (2011). 
7. Cook, J. A. \& Hawkins, D. B. Hearing loss and hearing aid treatment options. Mayo Clin Proc 81, 234237, doi:10.4065/81.2.234 (2006).

8. Ma, Y., Wise, A. K., Shepherd, R. K. \& Richardson, R. T. New molecular therapies for the treatment of hearing loss. Pharmacol Ther 200, 190-209, doi:10.1016/j.pharmthera.2019.05.003 (2019).

9. Omichi, R., Shibata, S. B., Morton, C. C. \& Smith, R. J. H. Gene therapy for hearing loss. Hum Mol Genet 28, R65-R79, doi:10.1093/hmg/ddz129 (2019).

10. Pauley, S., Kopecky, B., Beisel, K., Soukup, G. \& Fritzsch, B. Stem cells and molecular strategies to restore hearing. Panminerva Med 50, 41-53 (2008).

11. Sly, D. J. et al. Applying Neurotrophins to the Round Window Rescues Auditory Function and Reduces Inner Hair Cell Synaptopathy After Noise-induced Hearing Loss. Otol Neuroto/ 37, 1223-1230, doi:10.1097/MA0.0000000000001191 (2016).

12. Suzuki, J., Corfas, G. \& Liberman, M. C. Round-window delivery of neurotrophin 3 regenerates cochlear synapses after acoustic overexposure. Sci Rep 6, 24907, doi:10.1038/srep24907 (2016).

13. Wan, G., Gomez-Casati, M. E., Gigliello, A. R., Liberman, M. C. \& Corfas, G. Neurotrophin-3 regulates ribbon synapse density in the cochlea and induces synapse regeneration after acoustic trauma. Elife 3, doi:10.7554/eLife.03564 (2014).

14. Naeve, G. S. et al. Neuritin: a gene induced by neural activity and neurotrophins that promotes neuritogenesis. Proc Natl Acad Sci U S A 94, 2648-2653, doi:10.1073/pnas.94.6.2648 (1997).

15. Cantallops, I. \& Cline, H. T. Rapid activity-dependent delivery of the neurotrophic protein CPG15 to the axon surface of neurons in intact Xenopus tadpoles. Dev Neurobio/ 68, 744-759, doi:10.1002/dneu.20529 (2008).

16. Gao, R. et al. Exogenous Neuritin Promotes Nerve Regeneration After Acute Spinal Cord Injury in Rats. Hum Gene Ther 27, 544-554, doi:10.1089/hum.2015.159 (2016).

17. Di Giovanni, S. et al. Neuronal plasticity after spinal cord injury: identification of a gene cluster driving neurite outgrowth. FASEB J 19, 153-154, doi:10.1096/fj.04-2694fje (2005).

18. Liu, S. Y. et al. NRN1 and CAT Gene Polymorphisms, Complex Noise, and Lifestyles interactively Affect the Risk of Noise-induced Hearing Loss. Biomed Environ Sci 34, 705-718, doi:10.3967/bes2021.098 (2021).

19. Patel, M. \& Hu, B. H. MicroRNAs in inner ear biology and pathogenesis. Hear Res 287, 6-14, doi:10.1016/j.heares.2012.03.008 (2012).

20. Lee, R. C., Feinbaum, R. L. \& Ambros, V. The C. elegans heterochronic gene lin-4 encodes small RNAs with antisense complementarity to lin-14. Cel/ 75, 843-854, doi:10.1016/0092-8674(93)90529-y (1993).

21. Lagos-Quintana, M., Rauhut, R., Lendeckel, W. \& Tuschl, T. Identification of novel genes coding for small expressed RNAs. Science 294, 853-858, doi:10.1126/science.1064921 (2001).

22. Miranda, K. C. et al. A pattern-based method for the identification of MicroRNA binding sites and their corresponding heteroduplexes. Cell 126, 1203-1217, doi:10.1016/j.cell.2006.07.031 (2006). 
23. Friedman, R. C., Farh, K. K., Burge, C. B. \& Bartel, D. P. Most mammalian mRNAs are conserved targets of microRNAs. Genome Res 19, 92-105, doi:10.1101/gr.082701.108 (2009).

24. Ma, L., Yi, H. J., Yuan, F. Q., Guo, W. W. \& Yang, S. M. An efficient strategy for establishing a model of sensorineural deafness in rats. Neural Regen Res 10, 1683-1689, doi:10.4103/1673-5374.153704 (2015).

25. Hirose, K. \& Sato, E. Comparative analysis of combination kanamycin-furosemide versus kanamycin alone in the mouse cochlea. Hear Res 272, 108-116, doi:10.1016/j.heares.2010.10.011 (2011).

26. Rudnicki, A. et al. microRNA-224 regulates Pentraxin 3, a component of the humoral arm of innate immunity, in inner ear inflammation. Hum Mol Genet 23, 3138-3146, doi:10.1093/hmg/ddu023 (2014).

27. Ryan, A. F., Harris, J. P. \& Keithley, E. M. Immune-mediated hearing loss: basic mechanisms and options for therapy. Acta oto-laryngologica. Supplementum, 38-43,

doi:10.1080/00016480260094965 (2002).

28. Leijon, S. C., Peyda, S. \& Magnusson, A. K. Temporal processing capacity in auditory-deprived superior paraolivary neurons is rescued by sequential plasticity during early development. Neuroscience 337, 315-330, doi:10.1016/j.neuroscience.2016.09.014 (2016).

29. Avraham, K. B. et al. The noncoding genome and hearing loss. Hum Genet, doi:10.1007/s00439-02102359-z (2021).

30. West, B. A., Brummett, R. E. \& Himes, D. L. Interaction of kanamycin and ethacrynic acid. Severe cochlear damage in guinea pigs. Arch Otolaryngo/ 98, 32-37, doi:10.1001/archotol.1973.00780020036009 (1973).

31. Webster, M. \& Webster, D. B. Spiral ganglion neuron loss following organ of Corti loss: a quantitative study. Brain Res 212, 17-30, doi:10.1016/0006-8993(81)90028-7 (1981).

32. Ding, D., McFadden, S. L., Browne, R. W. \& Salvi, R. J. Late dosing with ethacrynic acid can reduce gentamicin concentration in perilymph and protect cochlear hair cells. Hear Res 185, 90-96, doi:10.1016/s0378-5955(03)00258-2 (2003).

33. Ding, D., McFadden, S. L., Woo, J. M. \& Salvi, R. J. Ethacrynic acid rapidly and selectively abolishes blood flow in vessels supplying the lateral wall of the cochlea. Hear Res 173, 1-9, doi:10.1016/s03785955(02)00585-3 (2002).

34. van Emst, M. G., Klis, S. F. \& Smoorenburg, G. F. Identification of the nonlinearity governing even-order distortion products in cochlear potentials. Hear Res 114, 93-101, doi:10.1016/s0378-5955(97)001561 (1997).

35. Asakuma, S. \& Snow, J. B., Jr. Effects of furosemide and ethacrynic acid on the endocochlear direct current potential in normal and kanamycin sulfate-treated guinea pigs. Otolaryngol Head Neck Surg (1979) 88, 188-193 (1980).

36. Ohtani, I., Ohtsuki, K., Omata, T., Ouchi, J. \& Saito, T. Potentiation and its mechanism of cochlear damage resulting from furosemide and aminoglycoside antibiotics. ORL J Otorhinolaryngol Relat Spec 40, 53-63, doi:10.1159/000275386 (1978). 
37. Lu, T. X. \& Rothenberg, M. E. MicroRNA. J Allergy Clin Immunol 141, 1202-1207, doi:10.1016/j.jaci.2017.08.034 (2018).

\section{Tables}

Table 1. Information about differentially expressed miRNAs. 
Co-up-regulated miRNAs Co-down-regulated miRNAs

\begin{tabular}{|c|c|c|}
\hline mmu-miR-6395 & mmu-miR-122-3p & mmu-miR-421-3p \\
\hline novel-mmu-miR208-3p & mmu-miR-9-3p & mmu-miR-132-3p \\
\hline mmu-miR-196a-5p & mmu-miR-153-3p & mmu-miR-128-2-5p \\
\hline novel-mmu-miR122-3p & mmu-miR-135a-5p & mmu-miR-297a-5p \\
\hline mmu-miR-365-3p & mmu-miR-9-5p & mmu-miR-297c-5p \\
\hline novel-mmu-miR202-3p & mmu-miR-128-3p & novel-mmu-miR327-5p \\
\hline novel-mmu-miR365-5p & mmu-miR-340-5p & mmu-miR-344c-3p \\
\hline mmu-miR-1247-3p & mmu-miR-376a-3p & mmu-miR-466i-5p \\
\hline mmu-miR-211-5p & mmu-miR-497a-5p & mmu-miR-466p-3p \\
\hline mmu-miR-3073a-5p & mmu-miR-185-5p & mmu-miR-466c-3p \\
\hline novel-mmu-miR13-5p & mmu-miR-384-5p & mmu-miR-466b-3p \\
\hline mmu-miR-181a-2-3p & mmu-miR-181c-5p & mmu-miR-181c-3p \\
\hline mmu-miR-133b-3p & mmu-miR-9b-3p & mmu-miR-34b-5p \\
\hline mmu-miR-21a-3p & mmu-miR-7b-5p & mmu-miR-1948-3p \\
\hline mmu-miR-93-3p & mmu-miR-129-5p & mmu-miR-466p-5p \\
\hline mmu-miR-145a-5p & mmu-miR-7a-1-3p & mmu-miR-491-5p \\
\hline mmu-miR-133a-3p & mmu-miR-487b-3p & mmu-miR-122b-3p \\
\hline mmu-miR-224-5p & mmu-miR-206-3p & mmu-miR-381-5p \\
\hline mmu-miR-1198-5p & mmu-miR-33-5p & mmu-miR-3099-3p \\
\hline mmu-let-7c-2-3p & mmu-miR-532-5p & mmu-miR-541-3p \\
\hline mmu-let-7a-1-3p & mmu-miR-381-3p & mmu-miR-183-3p \\
\hline novel-mmu-miR130-5p & mmu-miR-194-5p & mmu-miR-466c-5p \\
\hline mmu-miR-1247-5p & mmu-miR-598-3p & mmu-miR-532-3p \\
\hline \multirow[t]{5}{*}{ mmu-miR-339-5p } & mmu-miR-700-3p & mmu-miR-466b-5p \\
\hline & mmu-miR-344-3p & mmu-miR-466o-5p \\
\hline & novel-mmu-miR293-5p & mmu-miR-669h-5p \\
\hline & mmu-miR-7689-3p & mmu-miR-3093-3p \\
\hline & mmu-miR-122b-5p & mmu-miR-150-3p \\
\hline
\end{tabular}




\begin{tabular}{ll} 
mmu-miR-219a-5p & mmu-miR-669f-3p \\
\hline mmu-miR-129-2-3p & mmu-miR-6540-5p \\
\hline mmu-miR-212-3p & mmu-miR-201-5p \\
\hline mmu-miR-32-5p & mmu-miR-1188-5p \\
\hline mmu-miR-495-3p & novel-mmu-miR328-5p
\end{tabular}

\section{Figures}

A

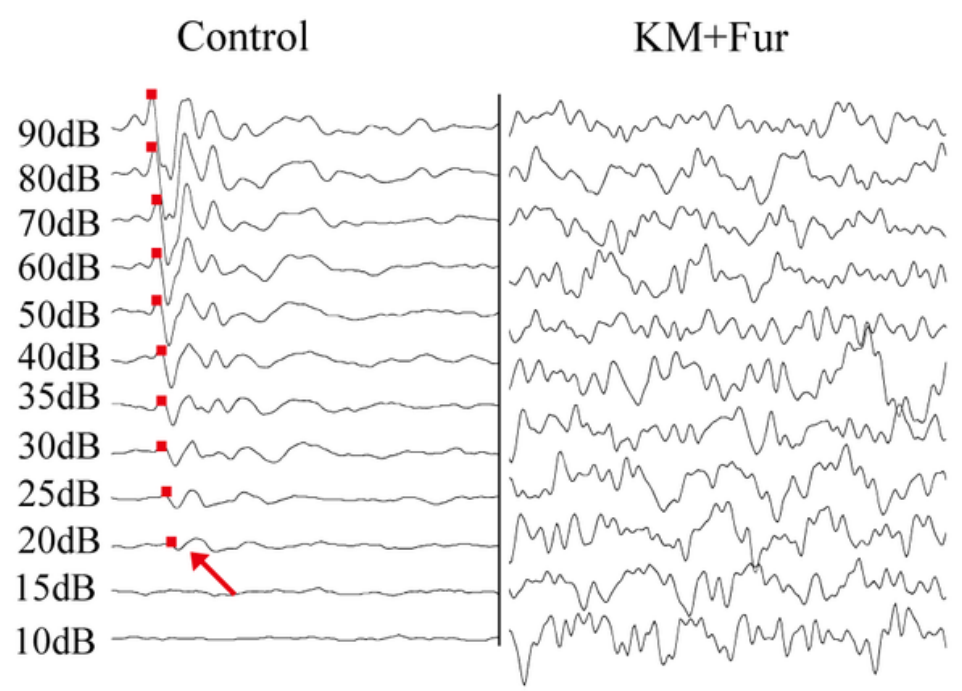

B

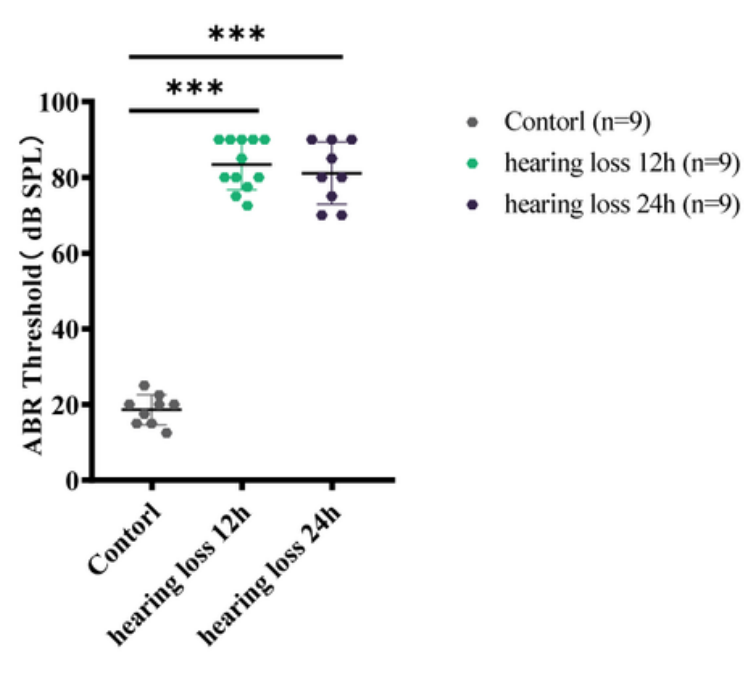

\section{Figure 1}

Hearing function detection results.

A: ABR waveform of mouse. B: Statistical analysis of ABR results.

Note: The red clipping head points to the hearing threshold judgment wave. 
A

B
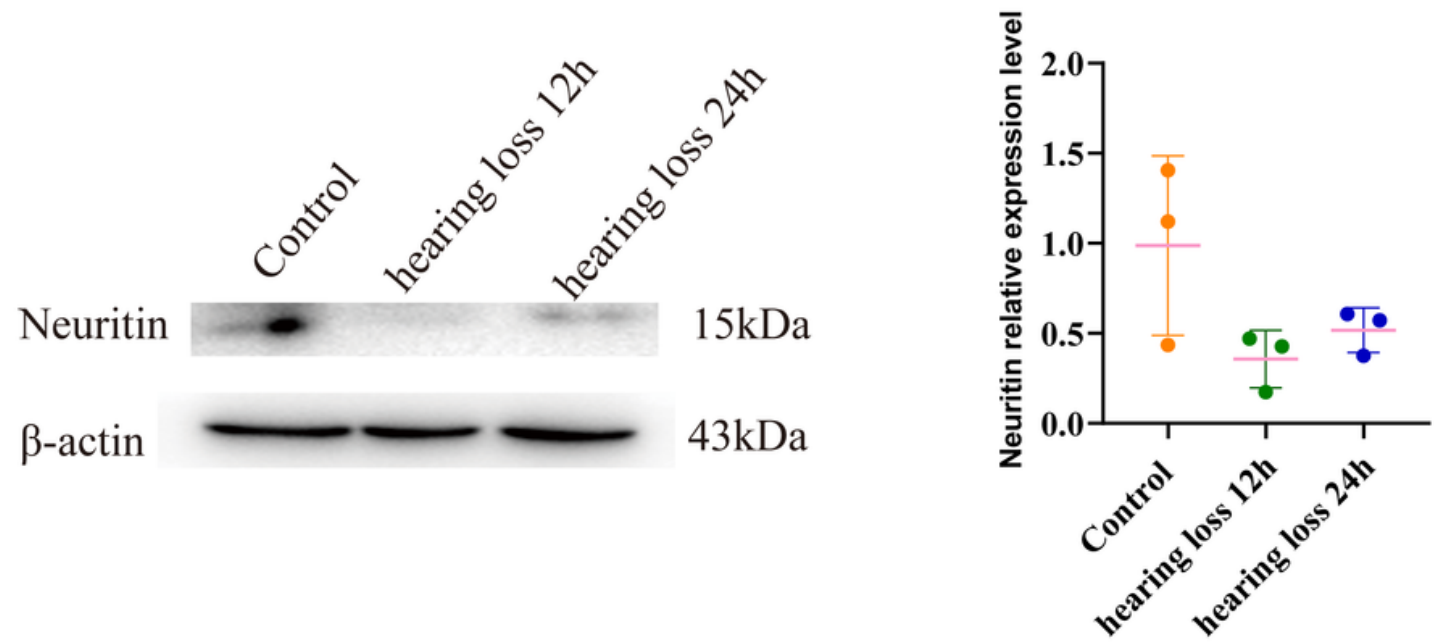

- Control $(n=3)$

- hearing loss $12 \mathrm{~h}(\mathrm{n}=3)$

- hearing loss $24 h(n=3)$

\section{Figure 2}

The relationship between hearing loss and Neuritin expression.

A: WB Results of Neuritin Expression Changes after hearing loss. B: Statistical analysis of Neuritin Expression Changes. 
A

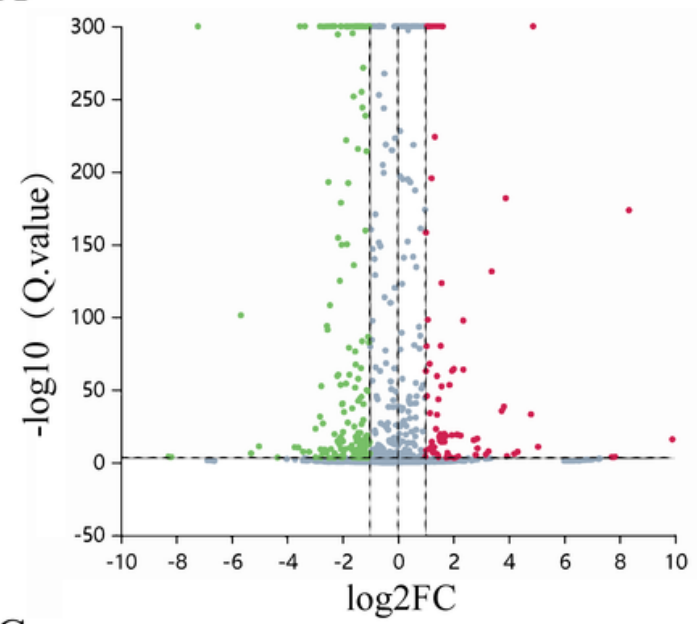

$\mathrm{C}$

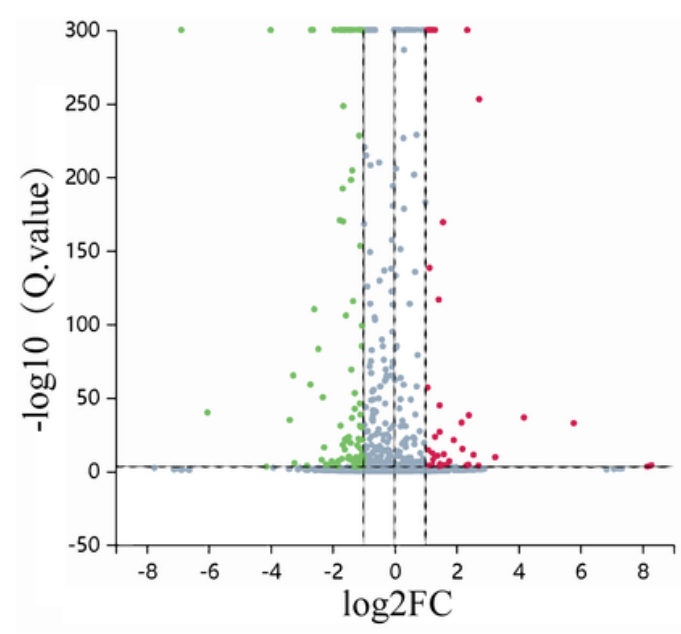

B
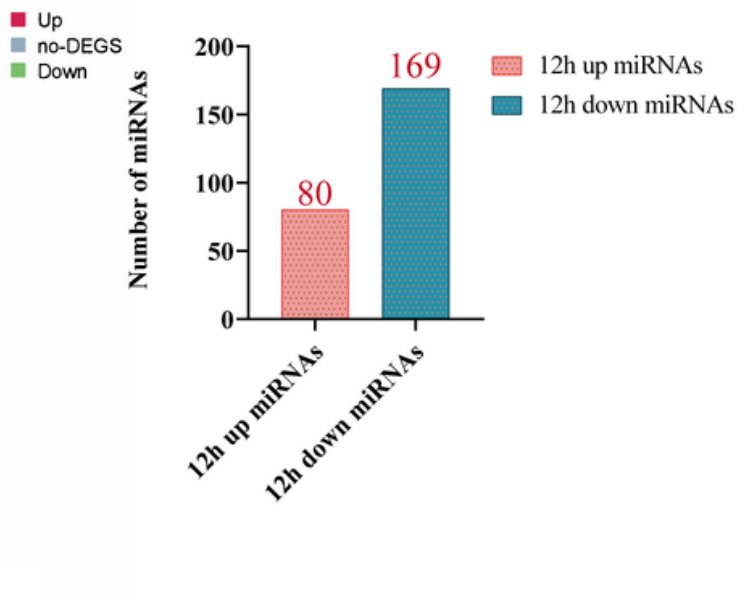

$\mathrm{D}$
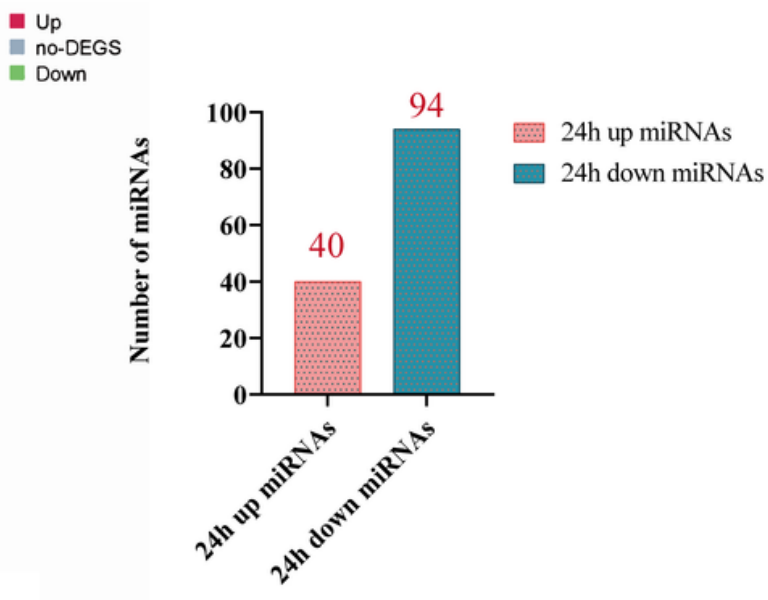

E

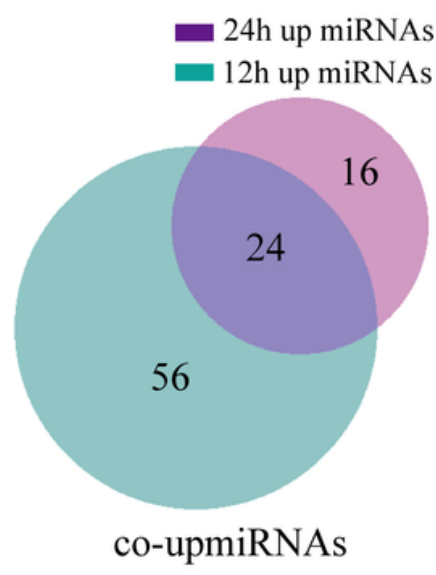

$\mathrm{F}$

24h down miRNAs

12h down miRNAs

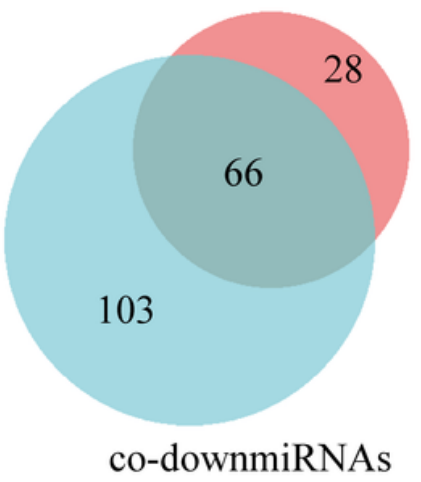

\section{Figure 3}

Screening difference expression miRNA

A: Difference miRNAs volcano illustration of $12 \mathrm{~h}$ group. B: Number of differential miRNAs in $12 \mathrm{~h}$ group. C: Difference miRNAs volcano illustration of $24 \mathrm{~h}$ group. D: Number of differential miRNAs in $24 \mathrm{~h}$ group. E: Co-upregulated miRNAs of 12 hours group and 24 hours group. F: Co-downregulated miRNAs of $12 \mathrm{~h}$ and $24 \mathrm{~h}$ group.

Note: Green dots represent declining miRNAs, red dots represent rising miRNAs, and grey dots represent discarded miRNAs. 


\section{A}

\section{miRNAs target Neuritin co-upmiRNAs}

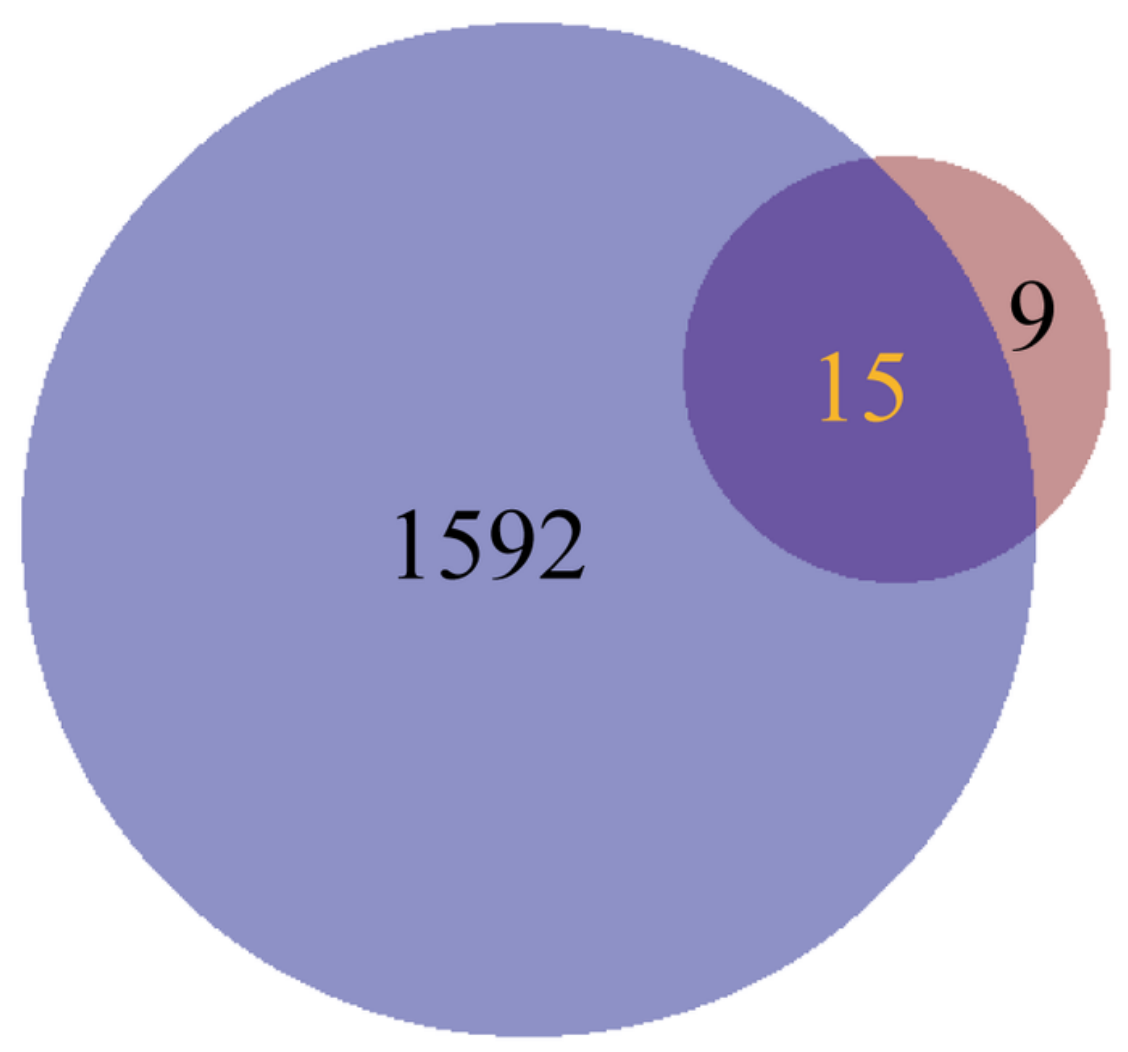

Figure 4

Screening miRNAs related to Neuritin and hearing loss

A: Overlap of Neuritin-targeting miRNAs and co-upregulated miRNAs in hearing loss.

A

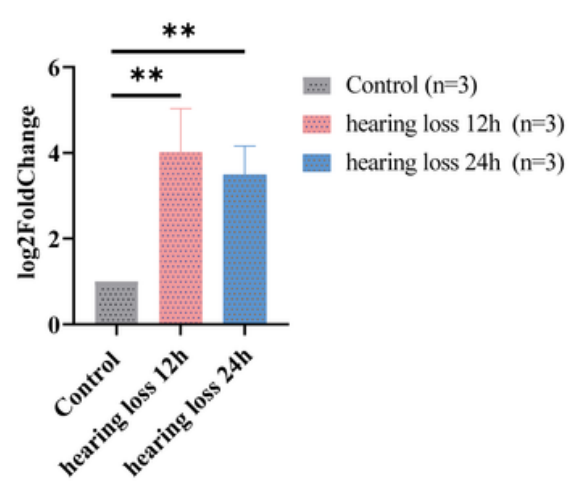

B

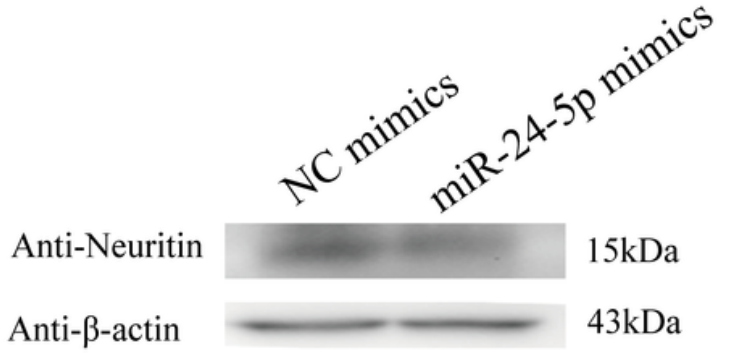

$\mathrm{C}$

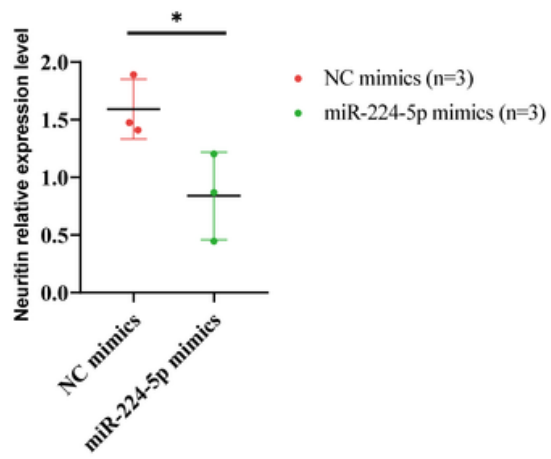


Figure 5

Correlation analysis of miRNA-224-5 with hearing loss and Neuritin

A: QRT-PCR results of miRNA-224-5p expression changes after hearing loss. B: WB result of Neuritin expression changes after transfected with miR-224-5p mimics. C: Statistical of Neuritn expression results.

A
3' UTR
NRN1 GAAAAGGAGAAGGCCAGGGGAAUGACUUCAAGAGUGG
mmu-miR-224-5p
UUGCCUUGGUGAUCACUGAAU

B

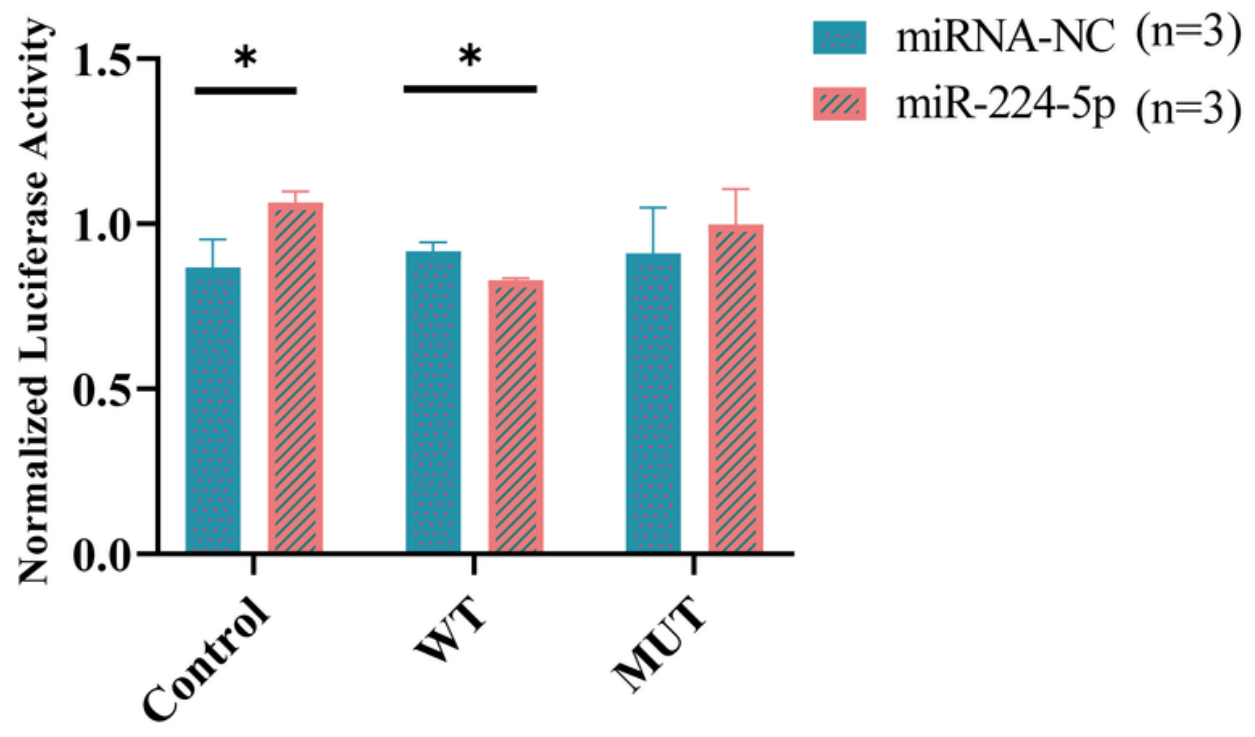

Figure 6

Analysis result of binding site between miR-224-5p and Neuritin

A: The prediction result of the binding site of miR-224-5p and Neuritin. B: Results of dual luciferase reporter gene for miR-224-5p and Neuritin.

\section{Supplementary Files}

This is a list of supplementary files associated with this preprint. Click to download. 
- F1.tif

- SupplementaryTableS1.xIsx

- SupplementaryTableS2.xIsx 